\title{
Attention and recollective experience in recognition memory
}

\author{
JOHN M. GARDINER \\ City University, London, England \\ and \\ ALAN J. PARKIN \\ University of Sussex, Brighton, England
}

\begin{abstract}
The functional relation between recognition memory and conscious awareness was assessed in an experiment in which undivided attention at study was compared with two divided attention conditions, one more demanding than the other. When recognizing a word from the study list, subjects indicated whether they could consciously recollect its prior occurrence or recognized it on some other basis, in the absence of conscious recollection. Divided attention at study progressively impaired word recognition accompanied by conscious recollection. Recognition in the absence of conscious recollection was not affected by divided attention. These findings are interpreted as providing further support for the idea that recognition memory entails two distinct components, one based on associative and contextual information, the other based on a "traceless" awareness of familiarity.
\end{abstract}

Recognition can be defined as what happens when an individual identifies a stimulus as having been encountered previously. Conscious awareness of recognition seems to take at least two distinct forms. A stimulus may evoke recognition when its occurrence brings to mind some specific experience in which the stimulus was previously involved. Alternatively, a stimulus may give rise only to feelings of familiarity. For example, one can recognize a person as being familiar without remembering who the person is, or without being able to recollect anything about the person.

A laboratory measure of these two kinds of conscious awareness is provided by Tulving's (1985b) distinction between "remember" and "know" responses. Word recognition measured by a "remember" response indicates that recognizing the word brings back to mind some conscious recollection of its prior occurrence in the study list, such as an association or image it triggered, something about its position or appearance, or something of more personal significance. Recognition measured by a "know" response indicates that recognizing the word brings nothing else to mind-no association, no contextual information, no conscious recollection of its prior occurrence in the study list.

Previous studies that have exploited these measures of conscious awareness in recognition memory have shown that they reflect distinct components in performance. Variables such as levels of processing, generate versus read, word frequency, and retention interval have influenced

Reprint requests should be addressed to John Gardiner, Memory \& Cognition Research Group, City University, Northampton Square, London ECIV OHB, England. only recognition accompanied by recollective experience, as measured by "remember" responses; recognition in the absence of recollective experience, as measured by "know" responses, has been uninfluenced by any of these variables (Gardiner, 1988; Gardiner \& Java, 1989, 1990).

Motivated by the striking similarity between variables that dissociate "remember" and "know" judgments and those that dissociate explicit and implicit memory phenomena more generally, Gardiner and Java (1990; see also Gardiner, 1988) have suggested that the distinction between "remember" and "know" responding can be interpreted within a theoretical framework (Hayman \& Tulving, 1989) that combines Tulving's $(1983,1985 \mathrm{a}$, 1985b) distinction between episodic memory and other memory systems with a processing account of differences between explicit and implicit measures of retention (Roediger \& Blaxton, 1987; Roediger, Weldon, \& Challis, 1989). In this framework, which is consonant with suggestions made by a number of other workers (Jacoby, 1983; Jacoby \& Dallas, 1981; Mandler, 1980, 1988; Richardson-Klavehn \& Bjork, 1988; Roediger et al., 1989; Schacter, in press; Tulving \& Schacter, 1990), " remember" responses reflect an episodic memory system that depends largely on conceptually driven processing, and on elaborative or contextual information. "Know" responses, in contrast, reflect some other "traceless" perceptual memory system that is primarily data-driven.

Gardiner and Java's (1990) hypothesis carries the clear implication that it should be possible to discover further parallels between recognition performance in the absence of conscious recollection and the known properties of implicit memory performance. One additional factor that has recently been shown to produce dissociations between implicit and explicit measures is the extent to which sub- 
jects are able to allocate conscious processing resources during initial learning. Parkin and Russo (1990) examined implicit memory performance by using savings measures in a picture-completion task. In their study, subjects were first exposed to fragmented picture sequences, which they were required to identify. While carrying out this task, half the subjects were also required to perform a secondary tone-monitoring task. Savings measures in picture completion were unaffected by secondary task performance, but, on a test of free recall for the pictures presented at study, the subjects in the tone-monitoring condition performed significantly worse. Parkin, Reid, and Russo (1990) carried out a similar manipulation, using a modification of the fragment-completion/recognition procedure of Tulving, Schacter, and Stark (1982). At presentation, subjects performed a sentence-classification task either undistracted or while monitoring tones. Primed word fragment completion was unaffected by tone monitoring, but it significantly impaired recognition performance. Related results have been reported by Jacoby, Woloshyn, and Kelley (1989), who showed that gains in familiarity, as measured by fame judgments of people's names from a previously presented list, were not reduced by a digitshadowing task that greatly reduced name recognition. These findings are consistent with the view that, unlike explicit memory performance, implicit memory performance does not depend on the amount of conscious processing resource available (see also Craik, 1989; Parkin, 1989; Stanhope \& Parkin, 1990).

Given the apparent similarity between the properties of explicit and implicit memory phenomena and those established for "remember" and "know" judgments, the present experiment was designed to test the hypothesis that dividing attention at study should selectively impair recognition accompanied by recollective experience, as measured by "remember" responses, but have little effect on recognition in the absence of recollective experience, as measured by "know" responses. There were three study conditions, one with undivided attention, and two with divided attention. The task chosen for dividing attention was the tone-monitoring task used by Parkin and Russo (1990) and Parkin et al. (1990). In the second divided attention task, the tones occurred at twice the rate used in the first condition, thus further reducing the amount of conscious processing resource available.

\section{METHOD}

\section{Subjects}

The subjects were 54 members of the Laboratory of Experimental Psychology subject panel at Sussex University; most of them were graduate students or technical and secretarial staff. They were allocated arbitrarily to one of three groups with 18 subjects in each. They were tested individually and were paid for their help. Three subjects were subsequently replaced, for apparently having failed to understand or act upon the recognition instructions.

\section{Design and Procedure}

Seventy-two target words were selected from the materials used by Tulving et al. (1982). These were divided into two study lists-
$\mathrm{A}$ and $\mathrm{B}-$ with 36 words in each. In the learning phase, the subjects sat in front of a visual display unit interfaced with a Motorola M6809 microprocessor. Half of the subjects in each condition were required to learn List $\mathrm{A}$, and the other half learned List $\mathbf{B}$. List items were presented individually at a rate of one every $2 \mathrm{sec}$. All subjects were instructed to attend to the words and try to remember them.

The subjects in the undivided attention condition studied the list undistracted. In the divided attention conditions, the subjects were required to listen to a tape-recorded tone sequence that comprised individual tones of low, medium, or high pitch. The pitch of any given tone in the sequence was randomly determined, and the presentation rate was quasirandom in that the time elapsing between any two successive tones varied randomly between a minimum and maximum time constraint. In the first divided attention condition, the tones occurred at intervals varying between 6 and $9 \mathrm{sec}$. The second condition was achieved by doubling the speed of the tape used in the first divided attention condition, thereby doubling the presen tation rate and halving the intervals between successive tones.

The subjects in the divided attention conditions were required to call out the pitch of each tone as it was presented ("high," "medium," or "low"), and they were given some initial training in this task. The subjects' responses were recorded by the experimenter. Performance on tone detection was good in both divided attention conditions. Only 19 out of 36 subjects made any errors at all; 10 of these made only one error.

Following list presentation, there was a 10 -min retention interval during which most subjects filled in a questionnaire about events spanning the last 30 years. The other subjects were engaged in genial conversation by the experimenter.

At recognition testing, the subjects were confronted with a single sheet comprising all 72 words used in both study lists; the words were arranged in four columns. The subjects were told to work down each column, indicating for each successive word whether or not they recognized it from the list that they had been shown earlier. In addition, for each word recognized, the subjects were asked to place the letter $\mathbf{R}$ by the word to indicate that they remembered it from the list or a $\mathrm{K}$ if they merely knew that the word was in the list. "Remember" and "know" responses were further explained as follows. The subjects were instructed to make "remember" responses to recognized words that evoked some specific recollection from the learning sequence. Examples given included remembering a word because it evoked a particular association, image, or some other more personal experience, or because something about its appearance or position could be recalled. "Know" responses were to be given for words that the subjects felt confident in recognizing but which failed to evoke any specific conscious recollection from the learning sequence. The distinction between the two responses was also illustrated by two more everyday examples. The subjects were told that if asked what movie they saw last, they would most probably "remember" because aspects of that experience would come back to mind; they were told that a "know" response was like recognizing someone in the street but not remembering who the person was, or being able to recollect anything at all about them. When subjects had completed the recognition phase, they were interviewed and asked to explain at least two of their "remember" judgments and two of their "know" judgments. This enabled the experimenter to establish whether the subjects had used the two types of response correctly.

\section{RESULTS}

The principal results of the experiment are shown in Figure 1. The figure indicates that divided attention conditions progressively impaired the level of correct "remember" responses but had no effect on the level of correct "know" responses. Separate analyses of variance 


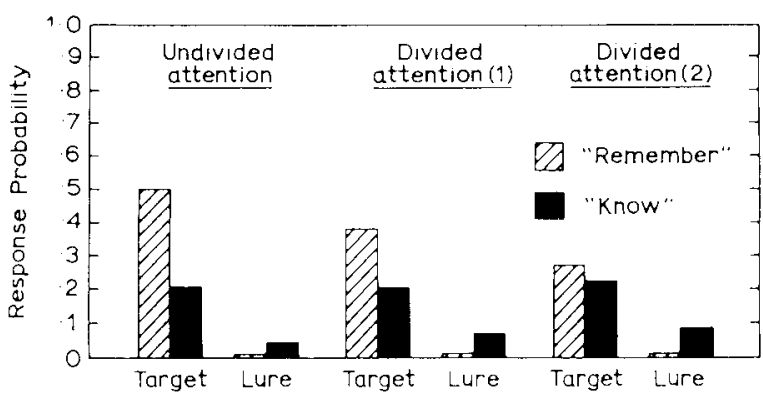

Figure 1. Response probability as a function of study conditions.

(ANOVA) for these "remember" and "know" responses confirmed this conclusion. ${ }^{1}$ For " "remember" responses, there was a significant main effect of study conditions $\left[F(2,53)=10.97, M S_{\mathrm{e}}=29.65, p<.001\right]$. Pairwise comparisons using a $t$ test for simple effects (Bruning \& Kintz, 1977) indicated that performance in all three conditions differed significantly from one another $(p<.01)$. A comparable ANOVA for "know" responses showed no significant effect of study conditions $[F<1$, $\left.M S_{\mathrm{e}}=11.88\right]$.

Examination of Figure 1 indicates a small rise in incorrect "know" responses across learning conditions, particularly in divided attention 2 . However, this apparent increase is mainly due to 1 subject in divided attention 2 who made an abnormally high number of incorrect "know" responses. This was confirmed by the results of an ANOVA, which revealed no significant effect of learning condition on incorrect "know" responses $[F=$ $1.28, M S_{\mathrm{e}}=9.053 \mathrm{]}$. The number of incorrect "remember" responses was too few for any meaningful statistical analysis.

The lower level of correct "know" responses overall might suggest that the absence of a main effect of learning condition was due to a floor effect. Two additional analyses rule out this interpretation. First, the standard errors for the three "know" conditions were, in the order undivided, divided attention 1 , divided attention $2, .03$, .03 , and .02 , indicating low variability around the performance means. Second, a median split analysis was carried out. This divided subjects into those producing a high or a low level of "know" responses. For the high-level group, the condition means were $.28, .27$, and .28 ; for the low-level group, they were $.13, .12$, and .16 . Together, these findings allow us to reject the possibility that failure to find an effect of learning condition on correct "know" responses arose from a floor effect.

\section{DISCUSSION}

The results of this experiment show that "remember" responses depend critically on the amount of conscious attention available during learning. This outcome supports the claim that "remembering" resembles other manifestations of explicit memory performance that are similarly influenced by divided attention (Craik, 1989; Jacoby, Woloshyn, \& Kelley, 1989; Stanhope \& Parkin, 1990). Conversely, the finding that "know" responses were not influenced by reductions in the amount of conscious processing resources available during learning supports the claim that "knowing" resembles other forms of implicit memory performance (Parkin et al., 1990; Parkin \& Russo, 1990; see also Jacoby, Woloshyn, \& Kelley, 1989).

This pattern of results is fully consistent with the hypothesis that "remember" responses indicate elaborative or conceptually driven processing in an episodic memory system, and that "know" responses indicate datadriven processing, or a "traceless" awareness of familiarity, arising in a perceptual representation system that does not include associative or semantic information (Schacter, in press; Tulving \& Schacter, 1990). By the same token, the results of the present experiment, and the other similar dissociations that have been observed between "remember" and "know' responses, clearly support the view that recognition memory entails two qualitatively distinct components, one of which may also be involved in implicit memory performance (see, e.g., Jacoby \& Dallas, 1981; Mandler, 1980).

Instead of reflecting components of memory qualitatively, however, it is logically possible that "remember" and "know" responses correspond to high and low degrees of confidence, or strong and weak trace strength. This possibility was investigated by Gardiner and Java (1990). They examined the pattern of "remember" and "know" responses for both word and nonword targets presented in a single study list (cf. Johnston, Dark, \& Jacoby, 1985). Gardiner and Java (1990) found a higher level of correct "know" responses for nonwords than for words. However, this increase in "know" responses to nonwords did not correspond with a lowering in measures of subjects' confidence. On the contrary, these higher levels of correct "know" responses corresponded with an increase in subjects' confidence that their responses were correct.

The present data provide additional evidence that "remember" and "know" responses do not correspond to different levels of subjective confidence or trace strength. It might be argued that there was a shift from high to low confidence following divided attention conditions. But if that were so, one would expect the divided attention conditions to generate a significant increase in the proportion of " know" responses to both targets and lures. Alternatively, if "know" and "remember" responses reflected a continuum of trace strength, then the proportion of "know" responses should decrease in the divided attention conditions. Neither of these predictions was supported.

This discussion leads us to consider alternative explanations of the relation between "remember" and "know" responses. The responses are defined by the task in a way that makes them mutually exclusive. This implies that correct recognition can be accompanied by only the one or 
the other measured kind of conscious awareness. However, as Jones (1987) has pointed out in another context, exclusivity is only one among several possible ways in which processes underlying memory performance may be related. The other possibilities he discussed are independence and redundancy.

Independence is the most widely adopted assumption in theoretical models proposing that two or more memory processes can give rise to the same overt outcome (see, e.g., Mandler, 1980). Applied to "remember" and "know" components, independence means that an item may be in one of four possible states, "remembered," "known," "remembered and known," or neither "remembered" nor "known." If, unlike the processing system assumed to give rise to "remember" responses, the processing system responsible for "know" responses is uninfluenced by an independent variable, then a relation of independence predicts that, as "remember" responses decrease, "know" responses should increase. This prediction is based on the assumption that, under normal circumstances, a proportion of recognized items would always fall into the "remembered and known" state. Neither the present experiment nor previous similar experiments provide any evidence that variables that decrease the proportion of "remember" responses increase the proportion of "know" responses.

Redundancy defines a relation in which the successful outcome from one component is possible only if the outcome from the second component is also successful. Although not widely used theoretically (but see Jones, 1987), this assumption is highly plausible for the relation between "remember" and "know" components. Intuitively one might expect that all recognizable words are "known" and that it is a subset of these that are also "remembered." However, this relation, like independence, predicts that as "remember" responses decrease, "know" responses should increase.

This leaves exclusivity as the remaining possible relation between the components underlying "remember" and "know" responses. Exclusivity assumes that the underlying components have no relation with one another, so that the outcome of one component exerts no influence whatsoever over the other component. This type of relation is consistent with evidence that has identified a number of variables that affect the probability of "remember" responses but have no influence on the level of "know" responses. ${ }^{2}$

Interestingly, although exclusivity has not been a widely adopted assumption, Jones (1987) drew attention to Tulving and Colotla's (1970) view that primary and secondary memory might be related in this way, rather than by the more commonly assumed independence between those two systems. Jones also pointed out that it is generally possible to devise alternative theoretical models in which existing assumptions of independence can be replaced by assumptions of exclusivity. This point is of particular interest in the context of the present discussion, because most systems and process models of memory, in- sofar as they specify the relation between systems or processes, seem to assume independence rather than exclusivity. Tulving and Schacter's (1990) account of the perceptual representation system underlying implicit memory phenomena is an interesting exception, because it is assumed that operations involving that system take place "without any obligatory engagement of other memory systems."

A final question concerns the exact relation between the generation of a correct "know" response and the nature of implicit responding. How can "know" responses reflect some implicit aspect of performance, when they clearly involve a judgment of prior occurrence in an explicit, episodic memory context? Perhaps the most useful perspective for thinking about this problem is provided by the attributional view of memory advocated by Jacoby and his colleagues (e.g., Jacoby, 1988; Jacoby, Allan, Collins, \& Larwill, 1988; Jacoby, Kelley, Brown, \& Jasechko, 1989; Jacoby, Kelley, \& Dywan, 1989). This research shows that unconscious or unaware forms of memory may give rise to a variety of mental experiences, and to different kinds of response, depending on how the situation is defined. For example, prior exposure to a previously unencountered name can, when the task requires judgments of fame, lead to that name "becoming famous overnight." That is, there is then a greater likelihood of that name being judged the name of a famous person, as opposed to other comparable names to which subjects had not been previously exposed. Similarly, prior exposure to an auditorily presented sentence can, when the task requires judgments of loudness, lead to that sentence then being perceived as being louder than other comparable sentences that subjects had not previously heard, even though the objective loudness does not differ. In a similar way, when the task requires judgments of recognition, then quite unconscious forms of memory may give rise to the conscious experience of familiarity, or feelings of knowing - and hence judgments of recognition in the absence of conscious recollection.

\section{REFERENCES}

Bruning, J. L., \& Kintz, B. L. (1977). Computational handbook of statistics. Glenview, IL: Scott, Foresman

Craik, F. I. M. (1989). On the making of episodes. In H. L. Roediger III, \& F. I. M. Craik (Eds.), Varieties of memory and consciousness: Essays in honor of Endel Tulving (pp. 43-57). Hillsdale, NJ: Erlbaum.

GARDINER, J. M. (1988). Functional aspects of recollective experience. Memory \& Cognition, 16, 309-313.

GARDINER, J. M., \& JAVA, R. I. (1989, November). Measuring conscious awareness in recognition memory. Paper presented at the meeting of the Psychonomic Society, Atlanta.

GARDINER, J. M., \&AVA, R. I. (1990). Recollective experience in word and nonword recognition. Memory \& Cognition, 18, 23-30.

Hayman, C. A. G., \& Tulving, E. (1989). Is priming in fragment completion based on a traceless memory system? Joumal of Experimental Psychology: Leaming, Memory, \& Cognition, 15, 941-956.

JACOBY, L. L. (1983). Remembering the data: Analyzing interactive processes in reading. Journal of Verbal Learning \& Verbal Behavior, $22,485-508$.

JACOBY, L. L. (1988). Memory observed and memory unobserved. In 
U. Neisser \& E. Winograd (Eds.), Remembering reconsidered: Ecological and traditional approaches to the study of memory (pp. 145177). New York: Cambridge University Press.

Jacoby, L. L., Allan, L. G., Collins, J. C., Larwill, L. K. (1988). Memory influences subjective experience: Noise judgments. Journal of Experimental Psychology: Learning. Memory, \& Cognition, 14, 240-247.

JaCOBY, L. L., \& Dallas, M. (1981). On the relationship between autobiographical memory and perceptual learning. Joumal of Experimental Psychology: General, 110, 306-340.

Jacoby, L. L., Kelley, C., Brown, J., Jasechko, J. (1989). Becoming famous overnight: Limits on the ability to avoid unconscious influences of the past. Journal of Personality \& Social Psychology, 56, 326-338,

Jacoby, L. L., Kelley, C., \& Dywan, J. (1989). Memory attributions. In H. L. Roediger, III, \& F. I. M. Craik (Eds.), Varieties of memory and consciousness: Essays in honor of Endel Tulving (pp. 391422). Hillsdale, NJ: Erlbaum.

JacoBy, L. L., Woloshy, V., Kelley, C. (1989). Becoming famous without being recognized: Unconscious influences of memory produced by dividing attention. Jourmal of Experimental Psychology: General, 118, 115-125.

Johnston, W. A., Dark, V. J., Jacoby, L. L. (1985). Perceptual fluency and recognition judgments. Joumal of Experimental Psychology: Learning, Memory, \& Cognition, 11, 3-11.

JONES, G. V. (1987). Independence and exclusivity among psychological processes: Implications for the structure of recall. Psychological Review, 94, 229-235.

Mandler, G. (1980). Recognizing: The judgment of previous occurrence. Psychological Review, 87, 252-271.

MandleR, G. (1988). Memory: Conscious and unconscious. In P. R. Solomon, G. R. Goethals, C. M. Kelley, \& B. R. Stephens (Eds.), Memory: Interdisciplinary approaches (pp. 84-106). New York: Springer-Verlag.

PARKIN, A. J. (1989). The nature and development of implicit memory. In S. Lewandowsky, J. Dunn, \& K. Kirsner (Eds.), Implicit memory: Theoretical issues (pp. 231-240). Hillsdale, NJ: Erlbaum.

Parkin, A. J., ReID, T., \& Russo, R. (1990). On the differential nature of implicit and explicit memory. Memory \& Cognition, 18, 507-514.

PARKIN, A. J., \&usso, R. (1990). Implicit and explicit memory and the automatic effortful distinction. European Joumal of Cognitive Psychology, 2, $71-80$.

RichardSON-KLAVEHN, A., BJoRK, R. A. (1988). Measures of memory. Annual Review of Psychology, 39, 475-543.

RoEDIGER, H. L., III, \& Blaxton, T. (1987). Retrieval modes produce dissociations in memory for surface information. In D. Gorfein \& R. R. Hoffman (Eds.), Memory and cognitive processes: The Ebbinghaus Centennial Conference (pp. 349-379). Hillsdale, NJ: Erlbaum.

Roediger, H. L., III, Weldon, M. S., \& Challis, B. H. (1989). Explaining dissociations between implicit and explicit measures of retention: A processing account. In H. L. Roediger, III, \& F. I. M. Craik (Eds.), Varieties of memory and consciousness: Essays in honor of Endel Tulving (pp. 3-41). Hillsdale, NJ: Erlbaum.

SCHacter, D. L. (in press). Perceptual representation systems and implicit memory: Toward a resolution of the multiple memory systems debate. In A. Diamond (Ed.), Development and neural bases of higher cognitive function. New York: Annals of the New York Academy of Sciences.
STANHOPE, N., \& PARKIN, A. J. (1990). Source forgetting in young and elderly adults: A resource-based account. Manuscript submitted for publication

Tulving, E. (1983). Elements of episodic memory. New York: Oxford University Press.

Tulving, E. (1985a). How many memory systems are there? American Psychologist, 40, 385-398.

Tulving, E. (1985b). Memory and consciousness. Canadian Psychologist, 26, 1-12

Tulving, E., Colotla, V. A. (1970). Free recall of trilingual lists. Cognitive Psychology, 1, 86-98.

Tulving, E. \& Schacter, D. L. (1990). Priming and human memory systems. Science, 247, 301-306.

Tulving, E., Schacter, D. L., \& Stark, H. A. (1982). Priming effects in word-fragment completion are independent of recognition memory. Journal of Experimental Psychology: Learning, Memory. \& Cognition, 8, 336-342.

\section{NOTES}

1. This analysis of "remember" and "know" responses as separate dependent variables contrasts with previous studies in which response type was treated as a fixed factor (e.g., Gardiner, 1988). One can question the validity of combining two different response measures within a single analysis. However, to provide continuity with the previous studies, a combined analysis was carried out. This revealed main effects of response type $\left[F(1,34)=46.15, M S_{\mathrm{e}}=23.6, p<.001\right]$ and learning condition $\left[F(2,68)=7.4, M S_{\mathrm{e}}=19.3, p<.01\right]$, as well as a significant interaction between the fixed factors $[F(2,68)=9.63$, $\left.M S_{\mathrm{e}}=19.3, p<.001\right]$. A combined analysis therefore supports the conclusions derived from the two separate analyses.

2. In all the studies of the effects of independent variables on the pattern of "remember" and "know" responses (Gardiner, 1988; Gardiner \& Java, 1989, 1990), the analyses have been based on the absolute proportion of each response type. However, because these variables decrease performance overall, it might be argued that a more appropriate analysis would be based on the relative proportions of the two kinds of response. From this perspective, the present findings and those from previous studies could be interpreted as showing an increase in the relative proportion of "know" responses as overall performance declines. If the variables do result in a shift from "remember" to "know" responses, one would expect, at least on some occasions, to observe a significant increase in the absolute proportion of "know" responses as "remember" responses decline. However, all these experiments show that the absolute level of "know" responses remains invariant across manipulations that reduce the level of "remember" responses. It seems extremely unlikely that, under these varying experimental conditions, the shift from "remember" to "know" and the overall decrease in performance should combine to produce absolute proportions of "know" responses that are essentially identical. A far more plausible interpretation is that "know" responses are unaffected by these experimental manipulations and that this is why "know" responses remain invariant. An analysis in terms of relative proportions is therefore considered inappropriate.

(Manuscript received January 8, 1990; revision accepted for publication April 2, 1990.) 